\title{
إدارية التعلم الذاتي (Self Directed Learning) في تعلم مهارة الكلام \\ في مركز اللغات لتعليم اللغة العربية بجامعة مولانا مالك إبراهيم الإسلامية \\ الحكومية مالانج ومعهد عبد الرحمن بن عوف بجامعة مُمَّية مالانج
}

\author{
أنيس لطيفة الإنسانية \\ طلبة جامعة مولانا مالك إبراهيم الإسلامية الحكومية مالانج \\ البريد الإلكتروني: anyeslathifa@gmail.com
}

\begin{abstract}
Abstrak
The Management of Self Directed Learning in Speaking Skills in The Arabic Language Center at State Islamic University of Maulana Malik Ibrahim Malang and Ma'had Abdurrahman Bin Auf of University of Muhammadiyah Malang include planning, implementation and evaluation. And the Self Directed Learning that is intended in this research is the way in speaking learning.

The research questions in this research are: 1) How the planning of self directed learning in speaking skills at The Arabic Language Center and Ma'had Abdurrahman Bin Auf ?. 2) How the implementation of self directed learning in speaking skills at The Arabic Language Center and Ma'had Abdurrahman Bin Auf ?. 3) How the evaluation of self directed learning in speaking skills at The Arabic Language Center and Ma'had Abdurrahman Bin Auf ?. In this research, the method used is case study with qualitative approach. And a method of data collection by observation and interview.

The results of this research is the Management of Self Directed Learning in Speaking Skills in The Arabic Language Center at State Islamic University of Maulana Malik Ibrahim Malang and Ma'had Abdurrahman Bin Auf of University of Muhammadiyah Malang, there is an equal point in planning, implementation and evaluation. The equal point of planning in the composition of the plan for the activities of daily education means memorizing the vocabulary. And the implementation in the formation of the Arab environment, and encourage students in the practice of speaking the Arabic language, and the preference of students in
\end{abstract}


choosing the method of learning. And on the one hand is to establish oral test on each student.

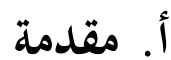

تعد الإدارة من أهم النشاطات الإنسانية التي تمارس داخل المجتمعات، حيث لها تأثير كبير على نواحي الحياة المختلفة نظراً لارتباطها المباشر بالشؤون الاقتصادية، والقضايا الاجتماعية، والسياسية. والإدارة هي عملية تحقيق الأهداف الموضوعة من خلال استغلال الموارد والعناصر المتاحة، حسب منهج مُحدّد، وضمن بيئة محددة، كما تعرف بأها عملية التخطيط والتنظيم، والرقابة، والتنسيق، والتوجيه المطبقة على الموارد المالية والبشرية، من أجل تحقيق أفضل النتائج، بأقل التكاليف، وأفضل الطرق وأقصرها،

$$
\text { والإدارة هي فرع من فروع العلوم الاجتماعية. ' }
$$

إن التعلم الذاتي هو محاولة إصلاح ضعف تدريس التقليدي. فمن جهة حاجة

المتعلمين، أن برنامج التعلم الذاتي أكثر فعالية لأن الطلاب يتعلم بنفسه، ومن جهة عمر المتعلمين أنه مناسب بالطلاب في مستوى العالي لأهم قد يستطيع في القراءة ويعمل الواجبة إما فرديا أو جماعة ويفهم الأمر الجيد، ومن جهة مادة الدراسة التي يمكن

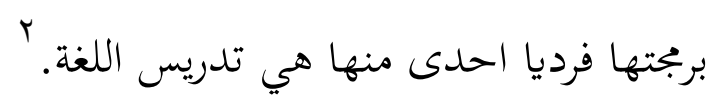

فمن الظواهر الموجودة، كان بعض الطلاب الذين يشتركون برنامج اللغة العربية ليس كلهم متخرجون في المعهد أو في المدرسة الإسلامية بحيث أفم لم يفهمون اللغة العربية جيدا. ولذلك قسمت لجنة البرنامج، الفصل حسب قدرة الطلاب في اللغة

\footnotetext{
2 Dimyati \& Mudjiono, Belajar dan Pembelajaran (Jakarta: Rineka Cipta, 2006), hal. 165
} 
العربية. وترى الباحثة أن الطالبات في الفصل لمستوى المبتدئ من فصل ج. بr في برنامج المكتف لتعليم اللغة العربية والفصل التمهدي في معهد عبد الرمن بن عوف بجامعة تُمَّمَية مالانج يطلبن الانتباه العميق في تعلم اللغة العربية، وبخاصة في مهارة الكلام. أكثرهن لم تستطيع التكلم باللغة العربية بالطلاقة والفصيحة بسبب نقصان تعريف المفردات وعدم استخدام اللغة العربية من قبل في اليومية.

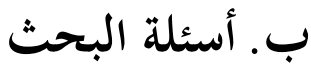

فبناء على مقدمة البحث التي شرحتها الباحثة فيما سبق، حددت

$$
\text { المشكلات لهذا البحث حول ما يلي: }
$$

1 - كيف تخطيط التعلم الذاتي في تعلم مهارة الكلام في مركز اللغات لتعليم اللغة

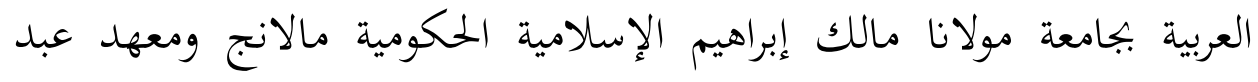

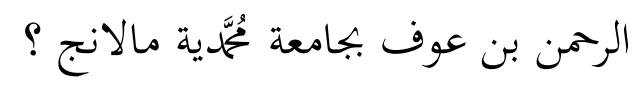

ץ- كيف تنفيذ التعلم الذاتي في تعلم مهارة الكلام في مركز اللغات لتعليم اللغة

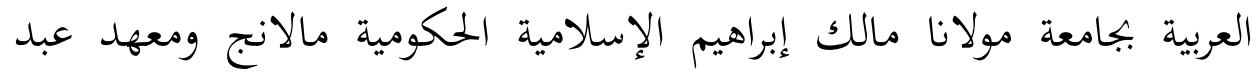

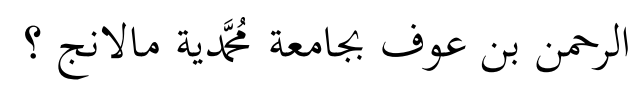

r- كيف تقويم التعلم الذاتي في تعلم مهارة الكلام في مركز اللغات لتعليم اللغة

العربية بجامعة مولانا مالك إبراهيم الإسلامية الحكومية مالانج ومعهد عبد

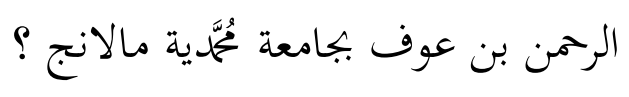


يهدف هذا البحث إلى أشياء مهمة بالنظر إلى أسئلة البحث السابق، وهي: 1- معرفة تخطيط التعلم الذاتي في تعلم مهارة الكلام في مركز اللغات لتعليم اللغة

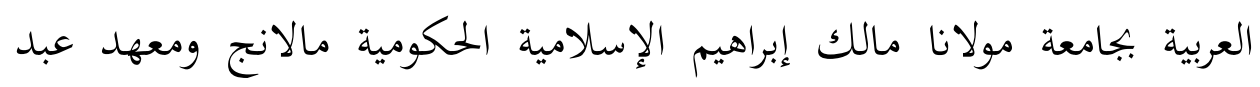
الرمن بن عوف بجامعة يُمَّمَية مالانج.

ץ- معرفة تنفيذ التعلم الذاتي في تعلم مهارة الكلام في مركز اللغات لتعليم اللغة ماته

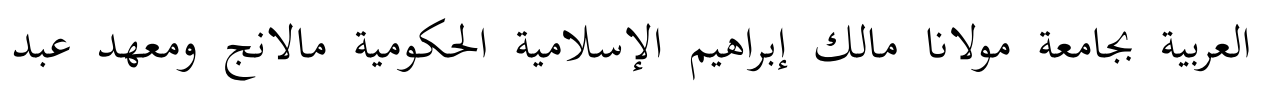
الرحمن بن عوف بجامعة يُجَّية مالانج. r- معرفة تقويم التعلم الذاتي في تعلم مهارة الكلام في مركز اللغات لتعليم اللغة

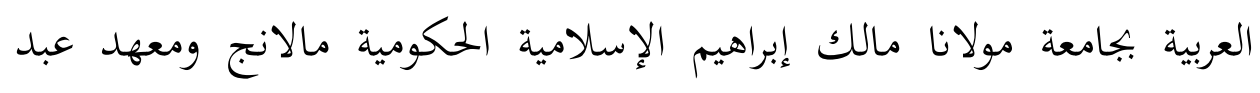
الرحمن بن عوف بحامعة عُمَّكَة مالانج.

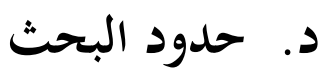

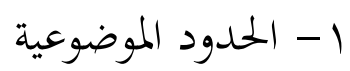

تحدد الباحثة عن تخطيط وتنفيذ وتقويم التعلم الذاتي في تعلم مهارة الكلام في مركز اللغات لتعليم اللغة العربية بجامعة مولانا مالك إبراهيم الإسلامية الحكومية مالانج ومعهد عبد الرمن بن عوف بجامعة مُحَّية مالانج. r- الحدود المكانية

موقع مكان هذا البحث العلمي في فصل ج. بrا، في مركز اللغات لبرنامج المكثف لتعليم اللغة العربية بجامعة مولانا مالك إبراهيم الإسلامية الحكومية 
مالانج وفي المستوى التمهيدي، في معهد عبد الرحمن بن عوف بجامعة مُحَّة

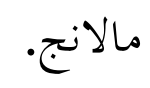

$$
\text { ب- الحدود الزمانية }
$$

تبدأ الباحثة القيام بالبحث العلمي في المستوى الأول.

\section{هـ. تحديد المصطلحات}

ليجتنب على سوء التفاهم والاحتياط على تفسيرات مختلفة لهذا الموضوع

$$
\text { فلازم على الباحثة أن تفهم محا يلي: }
$$

1- الإدارة : عملية إجتماعية تتضمن المسؤولية عن التخطيط الاقتصادي والتنظيم

$$
\text { الفعال لعملية المنظمة من أجل تحقيق هدف أو عمل محددا. }
$$

r- التعلم الذاتي: الطريقة التي تنظم برنامج التعلم في كل المادة الدراسية، منظم

بالطريقة المعينة الجاهزة لكل الطالب من أجل تسريع سرعة تعلمه تحت إشراف

$\varepsilon$ المعلم.

ب- مهارة الكلام: القدرة العالية على التحدّث، والحوار، والتعبير، أو إيصال فكرة، أو هدف لشخص أو مجموعة أشخاص أو جهة معيّنة. وذلك بامتلاك المضمون الجيد والأسلوب، كما يجب أن يمتلك المتحدّثث القدرة على التأثير والاقناع. وأن يتمتع بقدر عالٍ من الثقة في النفس ليكون كلامه أكثر قبولاً لدى الآخرين.

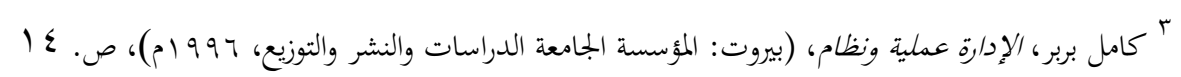

4 Joseph Mbulu, Pengajaran Individual Pendekatan, Metode, dan Media Pedoman Mengajar Bagi Guru dan Calon Guru, (Malang: Elang Emas, 2010), hlm. 1

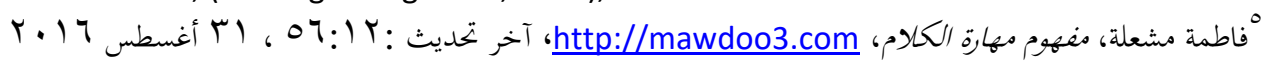




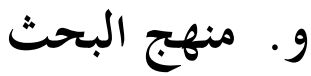

إن المنهج الذي استخدمت الباحثة هو دراسة الحالة بالمدخل الكيفي، وهذا يعني دراسة الحالة ببحث المتعددة. وكان ميدان البحث الذي اختارةا الباحثة هو مركز اللغات لبرنامج المكثف في تعليم اللغة العربية في جامعة مولانا مالك إبراهيم الإسلامية الحكومية مالانج ومعهد عبد الرمن بن عوف لكلية التربية الإسلامية في جامعة عمَّكَية مالانج. وأما مصدر البيانات تتكوّن من المدير والمدرّسة والطالبات. فتستخدم الباحثة الملاحظة والمقابلة لجمع البيانات. وتقوم الباحثة بعملية تحليل البيانات على طريقة ميليس وهوبيرمان(Miles \& Huberman) الذي يتكون من جمع البيانات، وعرض البيانات، ومناقشة البيانات وتحليلها.

\section{ز. ن نتائج البحث}

إن إدارية التعلم الذاتي في تعلم مهارة الكلام في مركز اللغات لبرنامج المكثف يشمل على التخطيط الذي يتكون من ملاحظ كفاءة الطالبة في عمل الواجبات اليومية ونتيجة التدريبات، ومساعدة الطالبات في تكلم اللغة العربية بالطلاقة، واختيار مصادر التعليم المناسب، وتعويد الطالبات لحفظ المفردات. وفي التنفيذ يتكون من تشجيع الطالبات في كل ممارسة الكلام، وتنفيذ أنشطة اللغوية، وتكوين البيئة اللغوية، تزويد الوسائل المحتاجة. وفي التقويم تراقب المعلمة حراكة الطالبات لكل الأفراد في خلال 
ممارسة الكلام أو في الاختبار، وتستخدم الحقائب التعليمية لتلخيص نتيجة الطالبات في خلال عملية التعليم والتعلم، وتقوم بالاختبار الشفهي. وإدارية التعلم الذاتي في تعلم مهارة الكلام في معهد عبد الرمن بن عوف تشمل على التخطيط الذي يتكون من تقديم السؤل إلى الطالبات عن أنماط التعلم ترغب فيها، وإقامة تدريب مهارة الكلام، وتقدر الطالبات التكلم باللغة العببية، واختيار مصادر التعليم المناسب، وذكر المفردات الجديدة. وفي التنفيذ يتكون من تمثيل الحوار، وتعويد قراءة النص العربية كثيرا، وتفضيل الطالبات لاختيار طريقة التعلم، وتكوين البيئة العربية، وتشجيع الطالبات في التكلم.وفي التقويم تدور المعلمة أثناء الطالبات غالبا، وتراقبها عند ممارسة الكلام أو في الاختبار. وتقوم الطالبات بالاختبار الشفهي. كان التعلم الذاتي في مركز اللغات لبرنامج المكثف لتعليم اللغة العربية بجامعة مولانا مالك إبراهيم الإسلامية الحكومية مالانج ومعهد عبد الرمن بن عوف بيامعة مُحََّية مالانج يهتم بأسس التعلم الذاتي، الأسس الفلسفية والأسس النفسية والأسس التربوية. وفي إدارية التعلم الذاتي، تلاحظ المعلمة أيضا على خصائص التعلم الذاتي وتستخدم أساليب التعلم الذاتي من خلال الحقيبة التعليمية، والتعلم بالحاسوب، والتعلم بلمراسلة بطريقة الوسائل السمعية البصرية لأجل مساعدة الطالبات في اكتساب مهارة اللغة العربية ولتكون ذاتيا في التعلم. 


\section{قائمة المراجع}

إبراهيم بسوني عميرة وفتحي الديب. تدريس العلوم والتربية العملية. الطبعة العاشرة. القاهرة:

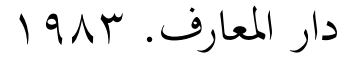

$$
\text { إبراهيم بن عبد العزيز. الإدارة العامة والإدارة التربية. عمان : الرواد. م ... }
$$

أحمد حامد منصور. التجاهات لتدريب الخلدمية في مجال النقنيات التربوية، وكيفية إعداد المتخصصين، والنهوض بالعاملين في هذا المجال فنيا وتربويا، لمستويات التعلم

المختلفة . مجلة تكنولوجيا التعلم. العدد الحادي عشر. الكويت: | 191 |

أحمد حامد منصور. التعلم الذاتي وكيفية إعداد برنامج تعليمي يكققه. بجلة تكنولوجيا

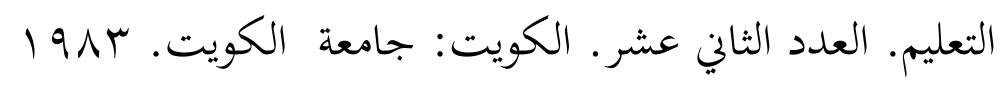

أحمد حسين اللقاني، وآخرون. تلدريس المواد الاجتماعية، الجز الثاني. القاهرة: علم الكتب.

199.

عبد الرحن عبد السلام جامل. التعلم الذاتي بالموديولات التعليمية. عمان: دار المناهج.

$$
1991
$$

http://mawdoo3.com تعريف الإدارة وأنواعها.

Administrator Ma'had. Panduan Akademik Ma'had Abdurrahman Bin 'Auf Fakultas Agama Islam. Malang: Universitas Muhammadiyah Malang. 2016

Dimyati \& Mudjiono. Belajar dan Pembelajaran. Jakarta: Rineka Cipta. 2006

Sugiyono. Metode Penelitian Pendidikan Kuantitatif, Kualitatif, $R \& D$. Bandung: Alfabeta. 2011 
Zainal Veitzhal Rival dkk. Islamic Management Meraih Sukses Melalui Praktek Manajemen Gaya Rasulullah secara Istiqomh. Yogyakarta: BPPB Anggota IKAPI 